\title{
Characterization of the Microbiome along the Gastrointestinal Tract of Growing Turkeys
}

\author{
Toby J. Wilkinson ${ }^{1 \dagger}$, A. A. Cowan ${ }^{1 * t}$, H. E. Vallin ${ }^{1}$, L. A. Onime ${ }^{1}$, Linda B. Oyama ${ }^{1}$, \\ S. J. Cameron ${ }^{1,2}$, Charlotte Gonot ${ }^{1}$, J. M. Moorby ${ }^{1}$, K. Waddams $^{1}$, V. J. Theobald ${ }^{1}$, \\ D. Leemans ${ }^{1}$, S. Bowra ${ }^{3}$, C. Nixey ${ }^{4}$ and Sharon A. Huws ${ }^{1 *}$ \\ ${ }^{1}$ Institute of Biological, Environmental and Rural Sciences, Aberystwyth University, Aberystwyth, United Kingdom, \\ ${ }^{2}$ Department of Surgery and Cancer, Faculty of Medicine, Imperial College London, London, United Kingdom, ${ }^{3}$ Phytatec \\ (UK) Ltd.-Plas Gogerddan, Aberystwyth, United Kingdom, ${ }^{4}$ British Poultry Council, London, United Kingdom
}

OPEN ACCESS

Edited by:

David Berry

University of Vienna, Austria

Reviewed by:

Timothy J. Johnson,

University of Minnesota, United States

Zhongtang Yu,

The Ohio State University Columbus,

United States

${ }^{*}$ Correspondence:

A. A. Cowan

syc@aber.ac.uk

Sharon A. Huws

hnh@aber.ac.uk

${ }^{\dagger}$ These authors have contributed equally to this work.

Specialty section

This article was submitted to Microbial Symbioses,

a section of the journal

Frontiers in Microbiology

Received: 07 February 2017

Accepted: 30 May 2017

Published: 22 June 2017

Citation:

Wilkinson $T J$, Cowan $A A$, Vallin $H E$ Onime LA, Oyama LB, Cameron SJ, Gonot C, Moorby JM, Waddams K, Theobald VJ, Leemans D, Bowra S,

Nixey $C$ and Huws SA (2017) Characterization of the Microbiome along the Gastrointestinal Tract of

Growing Turkeys.

Front. Microbiol. 8:1089.

doi: 10.3389/fmich.2017.01089
The turkey microbiome is largely understudied, despite its relationship with bird health and growth, and the prevalence of human pathogens such as Campylobacter spp. In this study we investigated the microbiome within the small intestine (SI), caeca (C), large intestine (LI), and cloaca (CL) of turkeys at 6, 10, and 16 weeks of age. Eight turkeys were dissected within each age category and the contents of the SI, C, LI, and CL were harvested. 16S rDNA based QPCR was performed on all samples and samples for the four locations within three birds/age group were sequenced using ion torrent-based sequencing of the $16 \mathrm{~S}$ rDNA. Sequencing data showed on a genus level, an abundance of Lactobacillus, Streptococcus, and Clostridium XI (38.2, 28.1, and 13.0\% respectively) irrespective of location and age. The caeca exhibited the greatest microbiome diversity throughout the development of the turkey. PICRUSt data predicted an array of bacterial function, with most differences being apparent in the caeca of the turkeys as they matured. QPCR revealed that the caeca within 10 week old birds, contained the most Campylobacter spp. Understanding the microbial ecology of the turkey gastrointestinal tract is essential in terms of understanding production efficiency and in order to develop novel strategies for targeting Campylobacter spp.

Keywords: turkey, 16S rDNA, microbiome, Campylobacter, gastrointestinal tract, small intestine, caecum, large intestine

\section{INTRODUCTION}

Poultry meat represents the main source of protein for human nutrition with consumption per capita being nearly twice that of red meat (Foley et al., 2011). Globally the US consumes the most poultry meat with the European Union following closely (average $22.2 \mathrm{Kg} /$ capita in 2006 for EU) (Magdelaine et al., 2008). Of this around $17 \%$ is attributable to turkey (Meleagris gallopavo) consumption, with chickens (Gallus gallus domesticus) being the main poultry consumed. The reason for poultry meat's popularity is attributed to leanness and lower price compared to most other meats.

The relationship between the gut microbiome of chickens, to bird health and efficient growth is well-known (Brisbin et al., 2008; Scupham et al., 2008; Yeoman et al., 2012; Danzeisen et al., 2013; Wei et al., 2013). Scupham et al. (2008) also showed that high density turkey production 
has altered the caecal microbiome of the turkey as compared with their wild counterparts. Recent next generation sequence-based data also shows that the gastrointestinal tract (GI) microbiome of turkeys is reasonably distinct to that found in chickens, with only 16-19\% similarity at a species level (Wei et al., 2013, 2016). It is also known that the turkey GI tract microbiome changes during the turkey growth phase (up to 7 weeks) but few studies expand further into maturity (Scupham, 2009; Danzeisen et al., 2015).

The prevalence of the food-poisoning bacterium Campylobacter spp. in poultry products is also a cause of major concern in terms of economic impact to the industry and human health (Silva et al., 2011). Campylobacter is the main cause of food-poisoning in developed countries, with 70,298 cases reported in the UK in 2011 (DEFRA, 2011). Nonetheless, this estimate is conservative as many cases are not reported (Tam et al., 2012). Campylobacter spp. and their effect on bird health is disputed, with many researchers believing that this genus causes minimal detrimental effect on the health of poultry. Nonetheless, once ingested by humans Campylobacter spp. cause diarrhea, abdominal pain and nausea which last between 5 and 7 days, with $10 \%$ of cases ending in hospitalization and $0.2 \%$ in death (MacRitchie et al., 2014; Thibodeau et al., 2015). It is also known that the infective dose required to cause illness in humans is only around 500 colony-forming units (Waag et al., 1999). Campylobacteriois in humans is normally associated with GI tract contamination of the poultry carcass during slaughter (Oakley et al., 2013; MacRitchie et al., 2014), and recent studies illustrate that up to $76 \%$ of carcasses in supermarkets have Campylobacter contamination at levels capable of causing illness (Skarp et al., 2016). Due to the predominant consumption of chicken, emphasis on understanding the chicken microbiome and specifically developing novel strategies to combat food poisoning linked to Campylobacter has focused on these birds, with much less emphasis on turkeys. However, Campylobacter spp. also inhabit the GI tract of other birds used for human consumption, including turkeys (Wei et al., 2013; Danzeisen et al., 2015; Skarp et al., 2016). It is assumed that Campylobacter spp. reside mainly within the caeca of turkeys akin to the situation in chickens, although few studies have investigated this using recently developed next generation sequencing, particularly early development of the birds to maturity.

The aims of this study were to assess bacterial diversity within the turkey small intestine, caeca, large intestine and cloaca at 6 , 10 , and 16 (slaughter age) weeks of age using next generation sequencing, coupled with quantitative PCR for detection of thermophilic Campylobacter spp. Increased understanding of the turkey microbiome, in particular Campylobacter spp., and colonization of the different parts of the turkey GI tract over time, will aid our understanding of turkey health and development of effective control interventions to limit cases of human Campylobacteriosis.

\section{MATERIALS AND METHODS}

\section{Study Design and Sample Harvesting}

All work described using animals was conducted in accordance with the requirements of the UK Animals (Scientific Procedures)
Act 1986 and with the approval of the Aberystwyth University Animal Welfare and Ethical Review Body. Turkeys were humanely euthanized using captive bolt by a registered license holder. Twenty-four female turkeys were obtained from a commercial producer at 5 weeks of age. The turkeys were subsequently reared in a broiler unit until 8 were slaughtered at each time point $(6,10$, and 16 weeks of age). Birds were fed a turkey grower mash (Table 1) (GLW Feeds Ltd, Loughborough, UK). All birds had constant access to fresh water. After slaughter the gastrointestinal tract of the turkeys were dissected and the contents from the whole small (SI) and large (LI) intestines were taken, whilst sub-samples of caeca (C), and cloacal (CL) material were taken and stored at $-20^{\circ} \mathrm{C}$ for assessment of the bacterial diversity and abundance as detailed below.

\section{DNA Extraction}

Genomic DNA was extracted from the turkey intestinal samples (10 mg fresh weight) using the BIO101 FastDNA ${ }^{\circledR}$ SPIN Kit for Soil (Qbiogene, Cambridge, UK) in conjunction with a FastPrep ${ }^{\circledR}$ cell disrupter instrument (Bio101, ThermoSavant, Qbiogene) according to the manufacturer's instructions with the exception that the samples were processed for $3 \times 30 \mathrm{~s}$ at speed 6.0 in the FastPrep instrument. DNA was quantified and qualityassured using the Epoch microplate spectrophotometer (Biotek, Bedfordshire, UK).

\section{S rDNA Ion Torrent PGM Sequencing}

$16 \mathrm{~S}$ rDNA ion torrent sequencing was completed for all GI tract locations for 3 birds within each of the age categories (6, 10 , and 16 weeks), resulting in 36 samples being sequenced in total. Only 36 sequences were sampled as this was the maximum that could be sequenced on the ion torrent chip, whilst providing the sequencing depth required. Amplicons of

TABLE 1 | Nutritional components of the turkey feed.

\begin{tabular}{lc}
\hline Ingredient & Part (\% of fresh feed) \\
\hline Wheat & 40.17 \\
Braz/Para hipro soya & 21.2 \\
Oat-X & 20 \\
Wheatfeed meal & 8 \\
Monogastric remix & 3 \\
Sunflower ext (36) & 3 \\
Dical phosphate (18\%) & 1.48 \\
Liquid lysine 50(T) & 0.68 \\
Limestone flour & 0.6 \\
DSM-ATL Turkey 2 & 0.5 \\
Methionine H-A liquid & 0.44 \\
Soya oil spray & 0.3 \\
Salt & 0.23 \\
Liquid fat & 0.2 \\
L-Threonine & 0.14 \\
Elancoban G200 (E:757) & 0.03 \\
Roxazyme G2G liquid 35.7\% & 0.03 \\
Natuphos 5000 liquid & 0.01
\end{tabular}


the V1-V2 variable region of the bacterial $16 \mathrm{~S}$ rDNA gene were generated in triplicate for each of the 36 samples by PCR using the primers 27F (5'AGAGTTTGATCMTGGCTCAG 3') and 357R (5' CTCCTACGGGAGGCAGCAG 3') followed by ion torrent sequencing using adaptors as described by Belanche et al. (2016). All PCR products were initially verified by electrophoretic fractionation on a $1.0 \%$ agarose gel for $1 \mathrm{~h}, 120 \mathrm{~V}$, and $80 \mathrm{MA}$ in $1 \%$ TAE (Tris base, acetic acid and EDTA) buffer before pooling of triplicate amplicons. The pooled PCR products $(30 \mu \mathrm{l}$ each sample) were subsequently run on a $2.0 \%$ agarose gel for $2 \mathrm{~h}, 120$ $\mathrm{V}$, and $80 \mathrm{MA}$ in $1 \%$ TAE buffer before bands were viewed and cut on a dark reader transilluminator (Clare Chemical Research, Colorado, USA). Amplicons were retrieved from cut bands using the Isolate II PCR and Gel Kit (Bioline, London, UK). Purified amplicons were verified and quantified using the Agilent High Sensitivity Assay Kit (Agilent Technologies, California, USA) prior to sequencing using the Ion Torrent PGM sequencer following the Ion PGM Template OT2 400 and Ion PGM HiQ Sequencing kits (Life Technologies Ltd, Paisley, UK). These sequences have been submitted to the short read archive in the NCBI database under accession number PRJEB14286.

\section{Quantitative PCR}

Total bacterial 16S rDNA QPCR were carried as described by Huws et al. (2013), Huws et al. (2014a,b), and Huws et al. (2016) for all samples generated $(8$ birds $\times 3$ ages $\times 4$ locations $=96$ samples). Campylobacter spp. were detected using a QPCR method developed by Lund et al. (2004), targeting 16S rDNA, and again for all 96 samples. Essentially, the reaction mixture $(25 \mu \mathrm{l})$ contained $1 \times$ POWER SYBR green PCR Master Mix (Applied Biosystems, Warrington, UK), $10 \mathrm{pmol}$ of each primer (campF2 - 5'-CACGTGCTACAATGGCATAT-3 and campR2 $5^{\prime}$-GGCTTCATGCTCTCGAGTT-3'), 10 pmol of the probe (campP2 - 5'-FAM-CAGAGAACAATCCGAACTGGGACABHQ1-3), and $2 \mu \mathrm{L}$ of template DNA (ca. $20 \mathrm{ng}$ ). Amplification for each QPCR involved $50^{\circ} \mathrm{C}$ for $2 \mathrm{~min}, 95^{\circ} \mathrm{C}$ for $10 \mathrm{~min}$, followed by 45 cycles of $95^{\circ} \mathrm{C}$ for $15 \mathrm{~s}$, followed by annealing at $58^{\circ} \mathrm{C}$ for $30 \mathrm{~s}$, extension at $72^{\circ} \mathrm{C}$ for $30 \mathrm{~s}$, with a final cycle of 5 min at $72^{\circ} \mathrm{C}$. All samples were run in duplicate. A Campylobacter spp. QPCR standard was prepared using DNA extracted from $C$. jejuni NCTC 11322.

\section{Taxonomy and Functional Gene Prediction}

Using the CD-HIT-OTU pipeline (Li et al., 2012) sequences were denoised, low quality sequences, pyrosequencing errors and chimeras were removed, then sequences were clustered into Operational Taxonomic Units (OTU's) at 97\% identity. OTU's containing fewer than 10 reads were excluded due to the likelihood of them being a sequencing artifact. OTUs were classified against the Greengenes 16S rRNA gene database (13.5) using MOTHUR (Schloss et al., 2009) and the taxonomy added to the OTU table. Phylogenetic Investigation of Communities by Reconstruction of Unobserved States 165 (PICRUSt) was used to predict the genomic and metabolic potential represented by the microbiota at each GI tract location in the different turkey ages. Using functions within the PICRUSt pipeline this was then normalized and used for metagenome inference of Kyoto
Encyclopedia of Genes and Genomes (KEGG) orthologs. The predicted functions (KOs) were then collapsed into hierarchical KEGG pathways using the categorize_by_function step in the PICRUSt pipeline.

\section{Statistical Analysis}

Principal component analysis ordination plots of OTU data were constructed using the Phyloseq program for R (McMurdie and Holmes, 2013). Taxonomical tables at phyla and genera (converted to \% of total reads) were subjected to analysis of variance (ANOVA) with sample as the fixed effect and blocking by turkey using GenStat (Payne et al., 2007). Interactions between GI location and turkey age were also investigated using ANOVA and phylum and genus level data. Calculations of alpha diversity and beta dispersion were performed using the phyloseq Bioconductor package in $\mathrm{R}$, multivariate ANOVA of braycurtis distance matrices were assessed by 1,000 permutations and corrected using the Bonferroni method (McMurdie and Holmes, 2013). QPCR data was also subjected to ANOVA with sample as the fixed effect and blocking by turkey using GenStat (Payne et al., 2007). Interactions between GI location and turkey age were also investigated using ANOVA for the QPCR data. Analysis of corrrelation between Campylobacter presence/abundance and microbiome composition was carried out at OTU and genus level using the Bioconductor package metagenomeSeq in R (Paulson et al., 2013). Statistical Analysis of Metagenomic Profiles (STAMP) was employed to analyse PICRUSt data. Samples were blocked by age and location, and subjected to ANOVA (multiple groups) with 1,000 permutations, Tukey-Kramer post-hoc analysis and corrected for multiple testing using the Bonferroni method. STAMP was further used to produce principle coordinate analysis (PCA) and extended error bar plots based on these analyses.

\section{RESULTS}

\section{Sequencing Data}

Post-quality control, we obtained a total of 4,485,560 reads of sequencing, averaging at 90,267reads/sample (Table 2). Average sequence length was 415 bp (Table 2).

\section{The Turkey Microbiome along the Gastrointestinal Tract}

The PCA plot based on OTUs showed that the microbiome of 10 week old birds was quite distinct to those of 6 and 16 week old birds irrespective of GI tract location (Figure 1). On a phyla level, and irrespective of GI tract

TABLE 2 | Sequencing information on average across sampling site and age.

\begin{tabular}{lc}
\hline Sequence information & Average bp (reads) \\
\hline Pre QC: Base pair (bp) count & $37,511,950(4,485,560)$ \\
Post QC: bp Count & $18,086,244(90,267)$ \\
Post QC: Sequence count & $502,396(10,351)$ \\
Post QC: Mean sequence length & $415(27)$
\end{tabular}


location and bird age, Firmicutes predominated (on average $84.5 \%$ of all sequence reads) (Figure 2, Table 3). Bacteroides, Actinobacteria, and Proteobacteria were the next predominating phyla after Firmicutes with an average abundance of 9.30, 4.11 , and $1.48 \%$ of total reads respectively (Figure 2, Table 3). On a phylum level, the main differences seen were that Bacteroidetes were more abundant in the caeca and firmicutes less abundant compared with other GI tract locations (Figure 2, Table 3). In terms of age, Bacteroidetes was lower in the GI tract of 10 week old birds, whilst Firmictues were higher compared with turkeys of 6 and 16 weeks of age (Figure 2, Table 3). Significant interactions between age and GI tract locations were also evident for the Bacteroidetes and Firmicutes (Table 3).

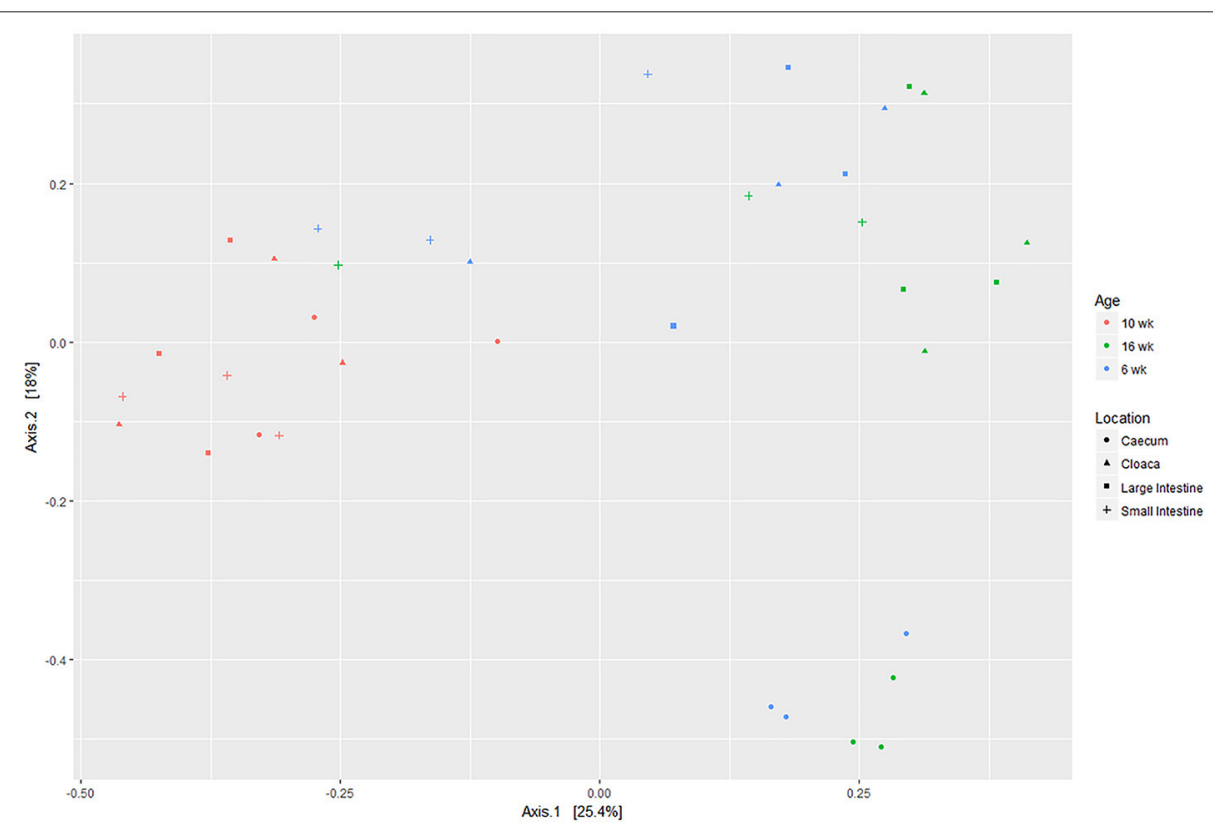

FIGURE 1 | Principal component analysis ordination plots generated using Phyloseq for R based on operational taxonomic units. Wk, week.

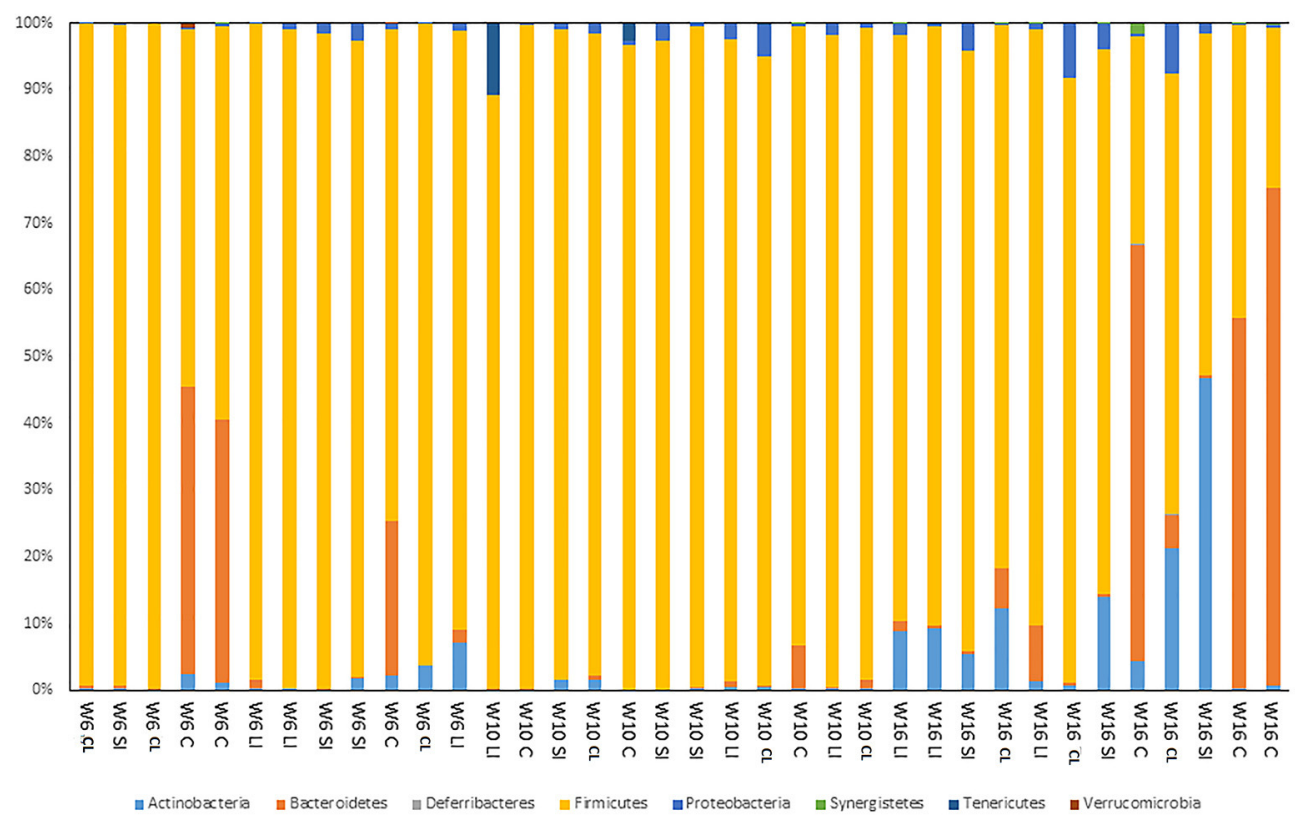

FIGURE 2 | Proportional representation of the turkey gut microbiome on a phylum level across the Gl tract of birds at 6, 10, and 16 weeks of age. W, Week; SI, Small intestine; LI, Large intestine; C, Caeca; CL, Cloaca gut microbiome. 
On a family/genus level, and irrespective of GI tract location and bird age, Lactobacillus, Streptococcus, and Clostridium XI predominated (38.2, 28.1, and 13.0\% respectively) (Figures 3, 4, Table 4, and Supplementary Tables 1-3). In terms of GI tract location, bacterial diversity in the small and large intestines were generally similar to each other in birds of all ages. The caecal bacterial diversity was highest in birds of all ages (Figures 3, 4, Table 4, Supplementary Figure 3 and Supplementary Tables 1-3). Alistipes, Anaerovorax, Bacteroides, Barnesiella, Blautia, Butyricicoccus, Campylobacter, Clostridium XIVb, Hallela, Paraprevotella, Phascolarctobacterium, Pseudoflavonifractor, Roseburia, Ruminococcus, Slackia, Subdoligranulum, Syntrophococcus, and unclassified bacteria were significantly $(P<0.05)$ higher in the caecum compared to the small and large intestine, whereas Streptococcus were significantly $(P<0.05)$ lower in abundance (Figures 3, 4, Table 4, and Supplementary Tables 1-3). Significant interactions between turkey age and GI tract location was seen for Alistepes, Anaerovorax, Bacteroides, Barnsiella, Howardella, Megaspahaera, Olsenella, Parabacteroides, Pelomonas, Ruminococcus, Slackia, Subdoligranulum, Syntrophococcus and unknown bacterial genera was seen (Table 4). The turkey cloacal microbiota showed most similarity to the microbiota within the large intestine, which is perhaps understandable given their close proximity (Figures 3, 4, Table 4, and Supplementary Tables 1-3). When considering the effect of turkey development on the GI

TABLE 3 | Bacterial phyla present within the small intestine, caecum, large intestine, and cloaca of mature turkeys.

\begin{tabular}{|c|c|c|c|c|c|c|c|c|c|c|c|c|}
\hline \multirow[t]{2}{*}{ Bacterial phylum } & \multicolumn{4}{|c|}{ Sample location } & \multicolumn{3}{|c|}{ Age } & \multicolumn{2}{|c|}{ SED } & \multicolumn{3}{|c|}{$P$} \\
\hline & SI & LI & C & $\mathbf{F}$ & 6 & 10 & 16 & Age & Sample & Age & Sample & Age ${ }^{\star S a m p l e}$ \\
\hline Actinobacteria & 7.80 & 3.10 & 1.30 & 4.50 & $1.60^{\mathrm{a}}$ & $0.40^{\mathrm{a}}$ & $10.40^{b}$ & 3.12 & 3.60 & 0.006 & 0.334 & NS \\
\hline Bacteroidetes & $0.20^{\mathrm{a}}$ & $1.60^{\mathrm{a}}$ & $33.90^{b}$ & $1.60^{a}$ & $9.20^{a b}$ & $0.80^{\mathrm{a}}$ & $17.90^{b}$ & 5.02 & 5.80 & 0.008 & $<0.001$ & $<0.001$ \\
\hline Deferribacteres & 0.00 & 0.00 & 0.05 & 0.00 & 0.00 & 0.00 & 0.04 & 0.04 & 0.05 & NS & NS & NS \\
\hline Firmicutes & $90.00^{b}$ & $93.00^{b}$ & $63.80^{a}$ & $91.30^{b}$ & $88.50^{b}$ & $96.20^{b}$ & $69.00^{a}$ & 5.19 & 5.99 & $<0.001$ & $<0.001$ & $<0.001$ \\
\hline Proteobacteria & $1.96^{a b}$ & $1.03^{a b}$ & $0.39^{a}$ & $2.55^{b}$ & $0.65^{a}$ & $1.36^{a b}$ & $2.44^{b}$ & 0.72 & 0.83 & NS & NS & NS \\
\hline Synergistetes & 0.00 & 0.01 & 0.26 & 0.01 & 0.02 & 0.00 & 0.19 & 0.11 & 0.12 & NS & NS & NS \\
\hline Tenericutes & 0.02 & 1.20 & 0.29 & 0.02 & 0.00 & 1.15 & 0.00 & 0.75 & 0.86 & NS & NS & NS \\
\hline Verrucomicrobia & 0.00 & 0.00 & 0.07 & 0.00 & 0.05 & 0.00 & 0.00 & 0.04 & 0.05 & NS & NS & NS \\
\hline
\end{tabular}

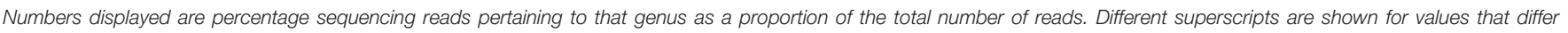
significantly from each other $(P<0.05)$.
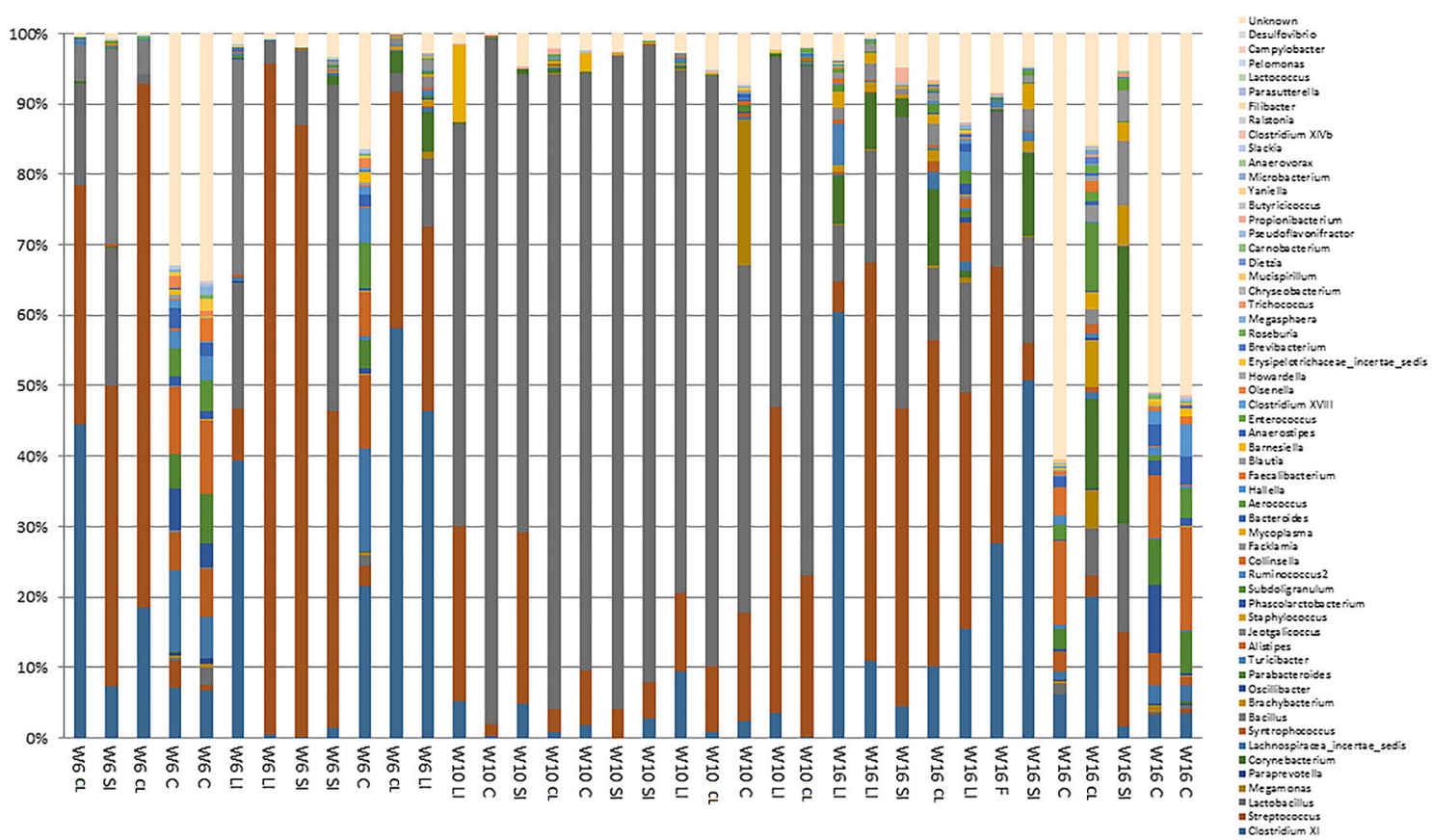

FIGURE 3 | Proportional representation of the turkey gut microbiome on a genus level across the Gl tract of birds at 6, 10, and 16 weeks of age. W, Week; SI, Small intestine; LI, Large intestine; C, Caeca; CL, Cloaca gut microbiome. 


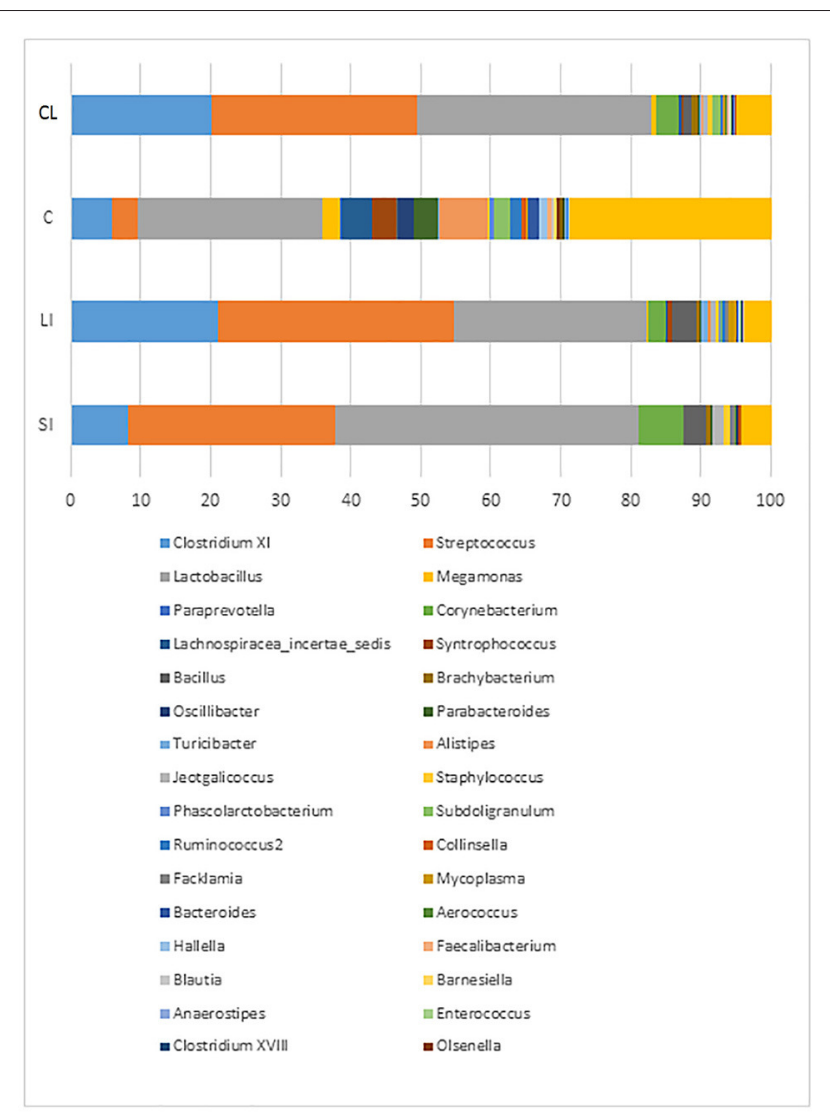

FIGURE 4 | Proportional representation of the turkey gut microbiome on a genus level on average for all turkey ages combined and grouped by the Gl tract location. SI, Small intestine; LI, Large intestine; C, Caeca; F, Cloaca gut microbiome.

tract microbiome, it is apparent that 10 week old birds show the most difference in their GI tract microbiome as a whole when compared to 6 and 16 week old birds (Figures 3, 4, Table 4, Supplementary Tables $1-3$ and Supplementary Figure 3). Ten-week-old turkeys commonly showed less diversity based on alpha diversity indices, when compared with 6 and 16 week old birds (Supplementary Figure 3). Ten week old turkeys had less Alistipes, Jeotgalicoccus, Parabacteroides, Phascolarctobacterium, and Streptococcus and more Campylobacter and Lactobacillus than 6 and 16 week old birds. Sixteen week old birds also had more Clostridium XI, Corynebacterium, Facklamia and unclassified bacteria than 6 and 10 week old birds (Figures 3, 4, Table 3, and Supplementary Tables 1-3). Estimates of beta diversity and dispersion suggested that the highly significant differences in diversity observed between age and location groups $(P<0.001)$ are not due to the variation in homogeneity between the groups $(P>0.1)$. Across the entire dataset, significant $(P<0.001$ and $P<0.01)$, moderate $(r=0.67$ and $r=0.49)$ correlations were seen between the presence/abundance of Campylobacter with Megamonas and Lactobacillus at both genus and OTU level.

\section{Turkey Microbiome Function}

PICRUSt data illustrated a range of potential functionalities (Supplementary Figure 1). ANOVA of PICRUSt data showed significant differences $(<0.05)$ in 11 KEGG pathways (Arginine and proline metabolism, cell division, energy metabolism, glycerolipid metabolism, methane metabolism, N-glycan biosynthesis, nitrogen metabolism, oxidative phosphorylation, pentose phosphate pathway, and transcription machinery) in the caecal microbiome when comparing 6 to 10 and 10 to 16 week old birds (all least abundant within 10 week old birds except RNA polymerase and transcription machinery which were at their lowest abundance in 6 week old birds), and two of these pathways (pentose phosphate and oxidative phosphorylation) also differed between 6 and 16 week old birds (pentose phosphate pathway was significantly higher in abundance within 6 week old birds compared with 16 week old birds and vice versa for gene abundances correlating to oxidative phosphorylation; Figure 5A). In the large intestine the D-arginine and the Dornithine pathway were significantly different in abundance when comparing 6 to 10 and 10 to 16 week old birds (highest abundances found in 10 week old birds; Figure 5B). In 6 week old birds metagenomic function differed when comparing the caeca to the large intestine and the caeca to the small intestine in 7 KEGG pathways (dioxin degradation, germination, $\mathrm{N}$-glycan biosynthesis and phenylpropanoid biosynthesis were higher in the caeca whereas Glycolysis/gluconeogenesis, phosphotransferase system (PTS) and secretion system gene abundances were lower in the caeca of 6 week old birds; Figure 5C), no significant differences were seen between the small and large intestines. In 16 week old birds comparing the caeca to the large intestine and the caeca to the small intestine highlighted 3 KEGG pathways significantly different (one carbon pool by folate was higher in the caeca, whereas gene abundances for other glycan degradation and others were higher in the SI of 16 week old birds; Figure 5D), again no differences were seen between the small and large intestines. Ten week old birds showed no significant differences in functionality along the GI tract. Principal coordinate analysis (PCA) of PC1 against PC2 showed some distinction in the function of the bacteria within 10 week old birds. In total PC1 accounts for $48.1 \%$ of the variability between samples and PC2 accounts for 31.4\% (Supplementary Figure 2).

\section{Total Bacterial and Campylobacter spp. 16S rDNA Quantitation}

Total bacterial 16S rDNA concentrations were higher in the caeca, with the highest concentrations found in the caeca of 16 week old birds (Table 5). Campylobacter spp. 16S rDNA concentrations were also higher in the caecum, with the highest concentrations found in the caeca of 10 week old birds (Table 5).

\section{DISCUSSION}

In this study we characterized the microbiome across the GI tract of maturing turkeys, with a specific focus on Campylobacter spp. Many studies have been completed on 
TABLE 4 | Bacterial genera present within the small intestine, caecum, large intestine, and cloaca of mature turkeys.

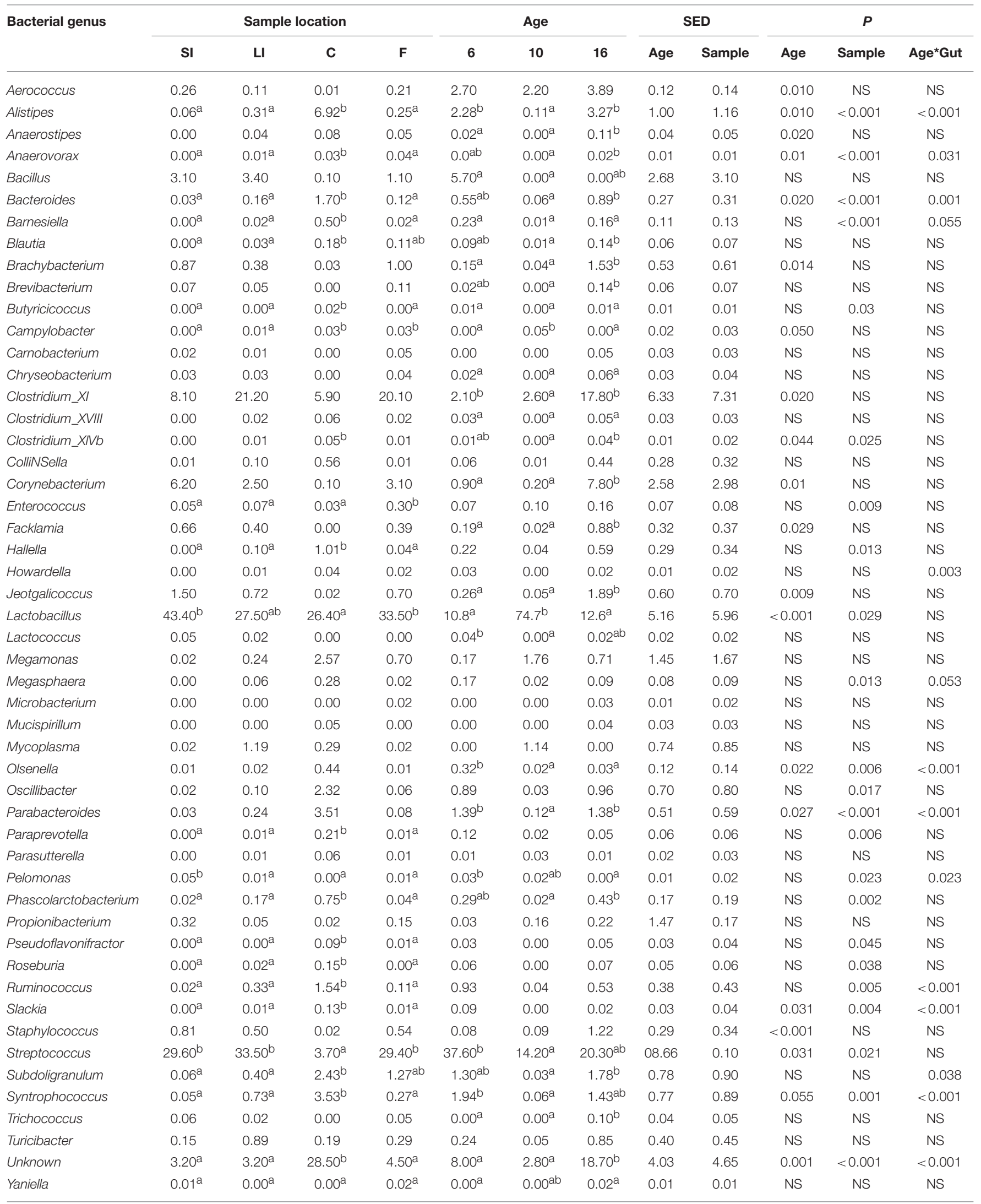

Numbers displayed are percentage sequencing reads pertaining to that genus as a proportion of the total number of reads. Different superscripts are shown for values that differ significantly from each other $(P<0.05)$. 


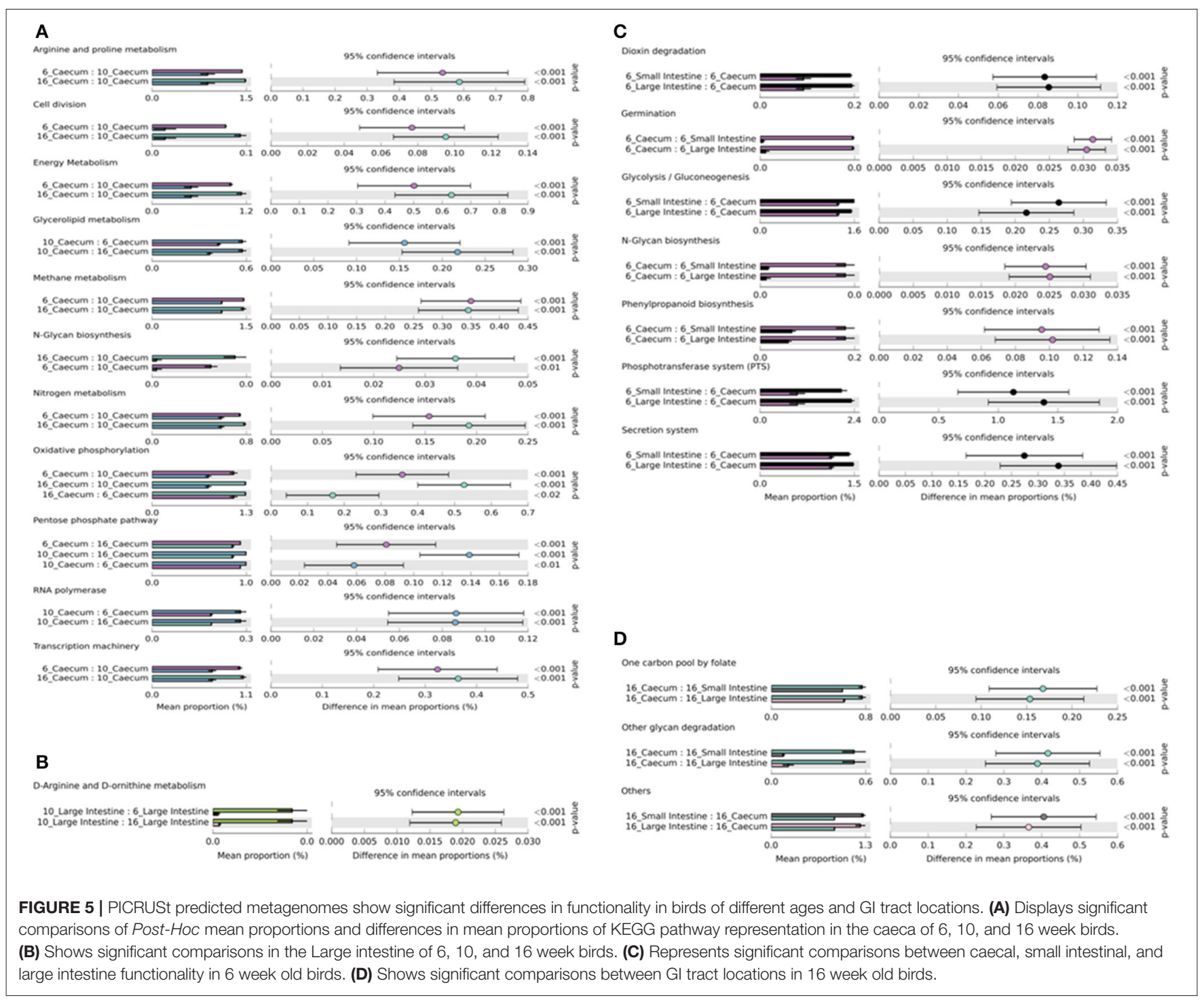

TABLE 5 | Effects of gastrointestinal tract location and turkey age on total bacterial and Campylobacter spp. 16S rDNA concentration.

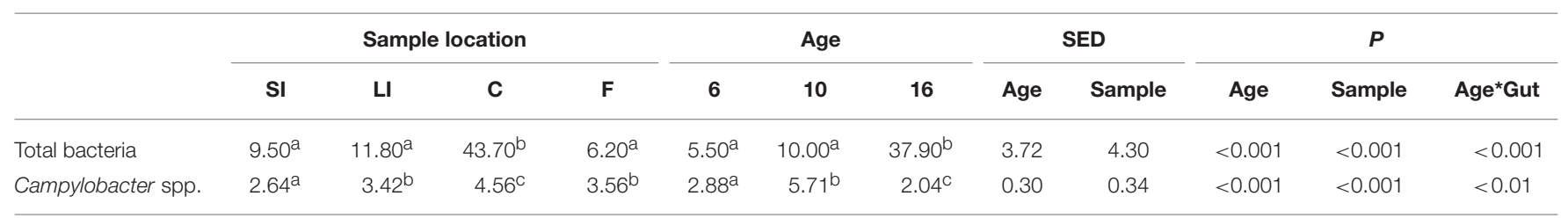

Numbers shown are $\log _{10} \mathrm{ng} / \mathrm{mg}$ sample dry matter. Different superscripts are shown for values that differ significantly from each other ( $\left.P<0.05\right)$.

the microbiome of chickens but fewer exist with respect to the turkey microbiome. We show that both GI tract location and turkey age have a significant effect on the whole gut microbiome present. We also show that Campylobacter spp. $16 \mathrm{~S}$ rDNA concentrations are most abundant within the caeca of 10 week old birds compared with 6 and 16 week old birds (Table 6). Understanding the turkey microbiome in various locations of the GI tract and over turkey maturation is crucial in order to understand production efficiency and also the pathogen load and risk with respect to human consumption.

On a phylum level, Firmicutes, Bacteroidetes, Actinobacteria, and Proteobacteria dominated within the microbiomes of the turkeys across age and GI tract location. This is in line with previously published metataxonomic data for the chicken and turkey gut microbiomes (Qu et al., 2008; Yeoman et al., 2012; Oakley et al., 2013; Wei et al., 2013; Choi et al., 2014; Mohd et al., 2015; Molina-Borda et al., 2016). In terms of GI tract location, 
TABLE 6 | Total bacterial and Campylobacter spp. 16S rDNA concentration in the small intestine (SI), large intestine (LI), caecum (C), and feces (f) of growing turkeys.

\begin{tabular}{|c|c|c|c|c|c|c|}
\hline & \multicolumn{4}{|c|}{ Sample location } & \multirow[t]{2}{*}{ SED } & \multirow[t]{2}{*}{$\boldsymbol{P}$} \\
\hline & SI & LI & C & $\mathbf{F}$ & & \\
\hline \multicolumn{7}{|c|}{ TOTAL BACTERIA } \\
\hline 6 weeks & $2.9^{\mathrm{a}}$ & $3.1^{\mathrm{a}}$ & $12.3^{b}$ & $2.6^{C}$ & 5.70 & $<0.001$ \\
\hline 10 weeks & $8.2^{\mathrm{ab}}$ & $13.0^{\mathrm{ab}}$ & $20.3^{b}$ & $4.4^{\mathrm{a}}$ & & \\
\hline 16 weeks & $15.0^{\mathrm{a}}$ & $18.3^{\mathrm{a}}$ & $97.5^{\mathrm{b}}$ & $12.7^{\mathrm{a}}$ & & \\
\hline \multicolumn{7}{|c|}{ Campylobacter spp. } \\
\hline 6 weeks & $2.3^{\mathrm{a}}$ & $2.5^{\mathrm{ab}}$ & $3.8^{C}$ & $3.0^{\mathrm{b}}$ & 0.57 & $<0.001$ \\
\hline 10 weeks & $3.7^{\mathrm{a}}$ & $5.5^{\mathrm{ab}}$ & $7.6^{\mathrm{C}}$ & $6.6^{b c}$ & & \\
\hline 16 weeks & $2.2^{\mathrm{a}}$ & $2.2^{\mathrm{a}}$ & $2.4^{\mathrm{a}}$ & $1.6^{\mathrm{a}}$ & & \\
\hline
\end{tabular}

Numbers shown are $\log _{10} \mathrm{ng} / \mathrm{mg}$ sample dry matter. Different superscripts are shown for values that differ significantly from each other $(P<0.05)$.

Bacteroidetes were significantly more abundant in the caeca, whilst the converse was true for Firmicutes, which were higher in abundance in the small and large intestine, and in cloaca material compared with caecal abundances. Proteobacteria predominated in the cloaca area, which is perhaps unsurprising as they are more tolerant of oxygen, which is likely to penetrate the cloaca and therefore be higher in abundance. Similar data have also been reported for the spatial nature of the broiler chicken microbiome (Choi et al., 2014; Mohd et al., 2015). When assessing the effect of age, on average Actinobacteria, Bacteroidetes, and Proteobacteria were more abundant in the GI tracts of 16 week old turkeys, whilst Firmicutes were higher in abundance in the GI tract of 6 and 10 week birds compared with 16 week birds.

On a genus/family level Lactobacillus, Streptococcus, and Clostridium_XI, dominated irrespective of gut location and turkey age. This data is in line with other reported data investigating the poultry gut microbiome to test differing hypotheses (Danzeisen et al., 2013, 2015; Choi et al., 2014; Videnska et al., 2014; Mohd et al., 2015; Oakley and Kogut, 2016). In terms of GI tract location, Alistipes, Bacteroides, Barnesiella, Butyricoccus, Clostridium_XIVb, Hallela, Paraprevotella, Phascolarctobacterium, Pseudoflavonifractor, Roseburia, Ruminococcus, Slackia, Syntrophococcus were higher in abundance in the caeca irrespective of turkey age. Blautia and Campylobacter had higher abundances in the caeca and cloaca than within the small and large intestines, whilst Anaerovorax and Corynebacterium dominated in the cloaca. Lactobacillus and Streptococcus had a significantly lower abundance in the caeca compared to abundances in the other GI tract locations. When assessing the effect of age, 10 week old birds generally showed the greatest difference in their GI tract microbiome as a whole when compared to 6 and 16 week old birds. Ten week old turkeys generally had less Alistipes, Jeotgalicoccus, Parabacteroides, Phascolarctobacterium, and Streptococcus and more Campylobacter and Lactobacillus than 6 and 16 week old birds. Sixteen week old birds also had more Clostridium XI, Corynebacterium, Facklamia and unclassified bacteria than 6 and 10 week old birds. Danzeisen et al. (2015) in a study investigating the ileal and caecal microbiome during maturation in 45 turkeys also noted that Clostridium XI increased in abundance in the GI tract as the birds aged, possibly as a consequence of their ability to ferment aromatic amino acids. Danzeisen et al. (2013) also noted that age was a key factor affecting the microbiome with Lactobacillus increasing in the ilea of turkeys as they age. We also found an increase in Lactobacillus in the SI in 10 week old birds compared with 6 week old birds, but numbers decreased within 16 week old birds. Alpha diversity indices showed that the caecal microbiome of 6 and 16 week old birds were higher in diversity compared with 10 week old birds.

Sequencing the rRNA gene of a gut microbiome is relatively simple and cost-effective, nonetheless understanding the function of the microbiome is key for understanding interrelationships with the host. Inferring function based on diversity of bacteria present can be difficult as the bacteria often transfer genes, and show a high degree of reliance and redundancy (Allison and Martiny, 2008). The relatively recently developed PICRUSt program has proved to be effective at obtaining functional predictions from 16S rRNA taxonomy data (Langille et al., 2013). Therefore, in an attempt to gain functional insight into the spatial and temporal function of the turkey gut microbiome we used PICRUSt. The main observations from the PICRUSt function data were that 10 week old birds differed significantly in the function of their caecal microbiome compared to birds of 6 and 16 weeks of age.

In this study we also show using next generation sequencing and QPCR that the abundance of thermophilic Campylobacter spp. in the turkey GI tract is at its highest within the caeca of 10 week old birds. It should also be noted that Campylobacter were underrepresented in our sequencing data compared to our QPCR data, despite the sequencing primers having a 100\% match to Campylobacter spp. Therefore, the reason for this cannot be determined although it is possibly a consequence of targeting secondary DNA structures in Campylobacter. Irrespective, in a previous study using pre-next generation sequencing technology, Scupham (2009) suggested that Campylobacter spp. vary with turkey age and are often linked with transition points within the whole microbiome diversity. Also, Thibodeau et al. (2015) suggested that levels of Campylobacter in the caeca of chickens were associated with changes in the microbiome particularly increasing in abundance when increases in Bifidobacterium, Mollicutes, and Clostria are seen. We saw a correlation with a rise in Campylobacter spp. abundance in the caeca and increases in Lactobacillus and Megamonas, whether they are causative linkages would need further investigation. Data for broiler chickens also suggests that post 8 weeks of age, the abundance of Campylobacter spp. in the caeca is reduced, which is hypothesized to be due to acquired immunity (Achen et al., 1998; Newell and Fearnley, 2003; Humphrey et al., 2014; Wigley, 2015; Reid et al., 2016). This raises the question that Campylobacter spp. are not natural commensals of the chicken gut, and also suggests that slaughtering at a later stage is potentially beneficial for human health. Our data for the turkey caecal microbiome also suggests that as turkeys reach slaughter age, acquired immunity may play a role in suppressing Campylobacter spp., although this hypothesis needs testing. 
These data highlight the complex interactions of the microbiome and the need to study the whole microbiome, and not the pathogens themselves in isolation.

In summary, in this study we show that the turkey gut microbiome, across the GI tract, changes in terms of taxonomy, diversity and function as the turkey matures with the main changes occuring in the caeca. We also show that Campylobacter reside predominantly in the caeca and numbers are higher at 10 weeks of age with reductions seen at age of slaughter. This study provides an understanding of the turkey gut microbiome, and contributes to the low number of publications available within the field as compared with chicken GI tract data. Understanding the microbial ecology of the turkey gastrointestinal tract is essential in terms of understanding production efficiency and in order to develop novel strategies for targeting Campylobacter spp.

\section{AUTHOR CONTRIBUTIONS}

Study idea and design: AC, JM, SB, CN, and SH; trial setup and sample collection HV, KW, and VT; sample analysis: TW, SC, LAO, LBO, and CG. Paper concept and writing: AC, TW, and

\section{REFERENCES}

Achen, M., Morishita, T. Y., and Ley, E. C. (1998). Shedding and colonization of Campylobacter jejuni in broilers from day-of-hatch to slaughter age. Avian Dis. 42, 732-737. doi: 10.2307/1592708

Allison, S. D., and Martiny, J. B. H. (2008). Resistance, resilience, and redundancy in microbial communities. Proc. Natl. Acad. Sci. U.S.A. 105(Suppl. 1), 11512-11519. doi: 10.1073/pnas.0801925105

Belanche, A., Jones, E., Parveen, I., and Newbold, C. J. (2016). A metagenomics approach to evaluate the impact of dietary supplementation with Ascophyllum nodosum or Laminaria digitata on rumen function in rustitec fermenters. Front. Microbiol. 7:299. doi: 10.3389/fmicb.2016. 00299

Brisbin, J. T., Gong, J., and Sharif, S. (2008). Interactions between commensal bacteria and the gut associated immune system of the chicken. Anim. Health Res. Rev. 9, 101-110. doi: 10.1017/S146625230800145X

Choi, J. H., Kim, G. B., and Cha, C. J. (2014). Spatial heterogeneity and stability of the bacterial community in the gastrointestinal tract of broiler chickens. Poultry Sci. 93, 1942-1950. doi: 10.3382/ps.2014-03974

Danzeisen, J. L., Calvery, A. J., Noll, S. L., McComb, B., Sherwood, J. S., Logue, C. M., et al. (2013). Succession of the turkey gastrointestinal bacterial microbiome related to weight gain. Peer J. 1:e237. doi: 10.7717/peerj.237

Danzeisen, J. L., Clayton, J. B., Huang, H., Kinights, D., McComb, B., Hayer, S. S., et al. (2015). Temporal relationships exist between cecum, ileum, and litter bacterial microbiomes in a commercial turkey flock, and subtherapeutic penicillin treatment impacts ileum bacterial community establishment. Front. Vet. Sci. 2:56. doi: 10.3389/fvets.2015.00056

DEFRA (2011). Zoonoses Report. London: DEFRA.

Foley, S. L., Nayak, R., Hanning, I. B., Johnson, T. J., Han, J., and Ricke, S. C. (2011). Population dynamics of Salmonella enterica serotypes in commercial egg and poultry production. Appl. Environ. Microbiol. 77, 4273-4279. doi: 10.1128/AEM.00598-11

Humphrey, S., Chaloner, G., Kemmet, K., Davidson, N., Williams, N., Kipar, A., et al. (2014). Campylobacter jejuni is not merely a commensal in commercial broiler chickens and affects bird welfare. MBio 5, e01364-e01314. doi: $10.1128 / \mathrm{mBio} .01364-14$

Huws, S. A., Edwards, J. E., Creevey, C. J., Rees Stevens, P., Lin, W., Girdwood, S. E., et al. (2016). Temporal dynamics of the metabolically active rumen bacteria
SH. All authors discussed the results and commented on the manuscript at all stages.

\section{ETHICS STATEMENT}

The study was conducted under a home office license.

\section{ACKNOWLEDGMENTS}

We acknowledge the sponsorship of the AD-Oat (HE 1116 1001-Added Value from Oats AD-Oat) project from European Regional Development Funding through the Welsh government's A4B Programme and support from our partners. Partners were the British Poultry Council, Phytatec, Oat Services, Poultry Xperience, and Bernard Matthews. IBERS also receives strategic funding from the BBSRC.

\section{SUPPLEMENTARY MATERIAL}

The Supplementary Material for this article can be found online at: http://journal.frontiersin.org/article/10.3389/fmicb. 2017.01089/full\#supplementary-material colonising fresh perennial ryegrass. FEMS Microbiol. Ecol. 92:e0149095. doi: $10.1093 /$ femsec/fiv137

Huws, S. A., Kim, E. J., Cameron, S. J. S., Girdwood, S. E., Davies, L., Tweed, J., et al. (2014a). Characterization of the rumen lipidome and microbiome of steers fed a diet supplemented with flax and echium oil. Microb. Biotechnol. 8, 331-341. doi: 10.1111/1751-7915.12164

Huws, S. A., Mayorga, O. L., Theodorou, M. K., Kim, E. J., Cookson, A. H., Newbold, C. J., et al. (2014b). Differential colonization of plant parts by the rumen microbiota is likely to be due to different forage chemistries. J. Microbiol. Biochem. 6, 080-086. doi: 10.4172/1948-5948.1000126

Huws, S. A., Mayorga, O. L., Theodorou, M. K., Onime, L. A., Kim, E. J., Newbold, C. J., et al. (2013). Successional colonization of perennial ryegrass by rumen bacteria. Lett. Appl. Microbiol. 56, 186-196. doi: 10.1111/lam. 12033

Langille, M. G. I., Zanevald, J., Caporaso, J. G., McDonald, D., Knights, D., Reyes, J. A., et al. (2013). Predictive functional profiling of microbial communities using 16S rRNA marker gene sequences. Nat. Biotechnol. 31, 814-821. doi: $10.1038 /$ nbt. 2676

Li, R. W., Wu, S., Li, W., and Li, C. (2012). Perturbation dynamics of the rumen microbiota in response to exogenous butyrate. PLoS ONE 7:e29392. doi: 10.1371/journal.pone.0029392

Lund, M., Nordentoft, S., Pedersen, K., and Madsen, M. (2004). Detection of Campylobacter spp. in chicken fecal samples by real-time PCR. J. Clin. Microbiol. 42, 5125-5132. doi: 10.1128/JCM.42.11.5125-5132.2004

MacRitchie, L. A., Hunter, C. J., and Strachan, N. J. C. (2014). Consumer acceptability of interventions to reduce Campylobacter in the poultry food chain. Food Control. 35, 260-266. doi: 10.1016/j.foodcont.2013. 06.005

Magdelaine, P., Spiess, M. P., and Valceschini, E. (2008). Poultry meat consumption trends in Europe. World's Poultry Sci. J. 64, 53-64. doi: $10.1017 /$ S0043933907001717

McMurdie, P. J., and Holmes, S. (2013). phyloseq: An R package for reproducible interactive analysis and graphics of microbiome census data. PLOS ONE 8:e61217. doi: 10.1371/journal.pone.0061217

Mohd, A. S., Asore, M., Sieo, C. C., Chong, C. W., Gan, H. M., and Ho, Y. (2015). Deciphering chicken gut microbial dynamics based on high-throughput 16S rRNA metagenomics analyses. Gut Pathog. 7:4. doi: 10.1186/s13099-0150051-7 
Molina-Borda, D., Vital, M., Sommerfeld, V., Rodehutscord, M., and CamarinhaSilva, A. (2016). Insights into broilers gut microbiota fed with phosphorus, calcium, and phytase supplemented diets. Front Microbiol. 7:2033. doi: $10.3389 /$ fmicb.2016.02033

Newell, D. G., and Fearnley, C. (2003). Sources of Campylobacter colonization in broiler chickens. Appl. Environ. Microbiol. 69, 4343-4351. doi: 10.1128/AEM.69.8.4343-4351.2003

Oakley, B. B., and Kogut, M. H. (2016). Spatial and temporal changes in the broiler chicken cecal and fecal microbiomes and correlations of bacterial taxa with cytokine gene expression. Front. Vet. Sci. 3:11. doi: 10.3389/fvets.2016.00011

Oakley, B. B., Morales, C. A., Line, J., Berrang, M. E., Meinersmann, R. J., Tillman, G. E., et al. (2013). The poultry-associated microbiome: network analysis and farm-to-fork characterizations. PLoS ONE 8:e57190. doi: 10.1371/journal.pone.0057190

Paulson, J. N., Stine, O. C., Bravo, H. C., and Pop, M. (2013). Differential abundance analysis for microbial marker-gene surveys. Nat. Methods 10, 1200-1202. doi: 10.1038/nmeth.2658

Payne, R. W., Murray, D. A., Harding, S. A., Baird, D. B., and Soutar, D. M. (2007). GenStat $^{\circledR}$ for WindowsTM, Introduction, 9th Edn. Hemel Hempstead: VSN International.

Qu, A., Brulc, J. M., Wilson, M. K., Law, B. F., Theoret, J. R., Joens, L. A., et al. (2008). Comparative metagenomics reveals host-specific metavirulomes and horizontal gene transfer elements in the chicken cecum microbiome. PLoS ONE 3:e2945. doi: 10.1371/journal.pone.0002945

Reid, W. D., Close, A. J., Humphrey, S., Chaloner, G., Lacharme-Lora, L., Rothwell, L., et al. (2016). Cytokine responses in birds challenged with the human foodborne pathogen Campylobacter jejuni implies a Th17 response. R. Soc. Open Sci. 3:150541. doi: 10.1098/rsos.150541

Schloss, P. D., Wescott, S. L., Ryabin, T., Hall, J. R., Hartmann, M., Hollister, E. B., et al. (2009). Introducing mother: open-source, platformindependent, community supported software for describing and comparing microbial communities. Appl. Environ. Microbiol. 75, 7537-7541. doi: 10.1128/AEM.01541-09

Scupham, A. J. (2009). Campylobacter colonization of the turkey intestine in the context of microbial community development. Appl. Environ. Microbiol. 75, 3564-3571. doi: 10.1128/AEM.01409-08

Scupham, J., Patton, T. G., and Bayles, D. O. (2008). Comparison of cecal microbiota of domestic and wild turkeys. Microb. Ecol. 56, 322-331. doi: 10.1007/s00248-007-9349-4

Silva, J., Leite, D., Fernandes, M., Mena, C., Gibbs, P. A., and Teixeira, P. (2011). Campylobacter spp. As a foodborne pathogen: a review. Front. Microbiol. 2:200. doi: 10.3389/fmicb. 2011.00200
Skarp, C. P., Hänninen, M.-L., and Rautelin, H. I. (2016). Campylobacterosis: the role of poultry meat. Clin. Microbiol. Infect. 22, 103-109. doi: 10.1016/j.cmi.2015.11.019

Tam, C. C., O’Brien, S. J., Adak, G. K., Meakins, S. M., and Frost, J. A. (2012). Campylobacter coli-an important foodborne pathogen. J. Infect. 47, 28-32. doi: 10.1016/S0163-4453(03)00042-2

Thibodeau, A., Fravolo, P., Yeargeau, E., Arsenault, J., Lahaye, L., and Letellier, A. (2015). Chicken caecal microbiome modifications induced by Campylobacter jejuni colonization and not by a nonantibiotic additive. PLoS ONE 10:e0131978. doi: 10.1371/journal.pone. 0131978

Videnska, P., Rahman, M. M., Faldynova, M., Babak, V., Matulova, M. E., PruknerRadovcic, E., et al. (2014). Characterization of egg laying hen and broiler fecal microbiota in poultry farms in Croatia, Czech Republic, Hungary and Slovenia. PLOS ONE 9:e110076. doi: 10.1371/journal.pone.0110076

Waag, A. S., Vardend, T., Lund, V., and Kapperud, G. (1999). Detection of small numbers of Campylobacter jejuni and Campylobacter coli cells in environmental water, sewage, and feed samples by a seminested PCR assay. Appl. Environ. Microbiol. 65, 1636-1643.

Wei, S., Lilburn, M., and Yu, Z. (2016). The bacteriomes of ileal mucosa and cecal content of broiler chickens and turkeys as revealed by metagenomics analysis. Int. J. Microbiol. 2016:12. doi: 10.1155/2016/4320412

Wei, S., Morrison, M., and Yu, Z. (2013). Bacterial census of poultry intestinal microbiome. Poultry Sci. 92, 671-683. doi: 10.3382/ps.2012-02822

Wigley, P. (2015). Blurred lines: pathogens, commensals, and the healthy gut. Front. Vet. Sci. 2:40. doi: 10.3389/fvets.2015.00040

Yeoman, C. J., Chia, N., Jeraldo, P., Sipos, M., Goldenfeld, N. D., and White, B. A. (2012). The microbiome of the chicken gastrointestinal tract. Anim. Health Res. Rev. 13, 89-99. doi: 10.1017/S1466252312000138

Conflict of Interest Statement: The authors declare that the research was conducted in the absence of any commercial or financial relationships that could be construed as a potential conflict of interest.

Copyright (c) 2017 Wilkinson, Cowan, Vallin, Onime, Oyama, Cameron, Gonot, Moorby, Waddams, Theobald, Leemans, Bowra, Nixey and Huws. This is an openaccess article distributed under the terms of the Creative Commons Attribution License (CC BY). The use, distribution or reproduction in other forums is permitted, provided the original author(s) or licensor are credited and that the original publication in this journal is cited, in accordance with accepted academic practice. No use, distribution or reproduction is permitted which does not comply with these terms. 